\title{
Theory of absorption-induced transparency
}

\author{
Sergio G. Rodrigo, ${ }^{1,2, *}$ F. J. García-Vidal, ${ }^{3}$ and L. Martín-Moreno ${ }^{2}$ \\ ${ }^{1}$ Centro Universitario de la Defensa, Carretera de Huesca s/n, E-50090 Zaragoza, Spain \\ ${ }^{2}$ Instituto de Ciencia de Materiales de Aragón and Departamento de Física de la Materia Condensada, \\ CSIC-Universidad de Zaragoza, E-50009 Zaragoza, Spain \\ ${ }^{3}$ Departamento de Física Teórica de la Materia Condensada and Condensed Matter Physics Center (IFIMAC), \\ Universidad Autónoma de Madrid, 28049 Madrid, Spain
}

(Received 27 July 2013; published 21 October 2013)

\begin{abstract}
Recent experiments [Hutchison, O’Carroll, Schwartz, Genet, and Ebbesen, Angew. Chem. Int. Ed. 50, 2085 (2011)] have demonstrated that optical transmission through an array of subwavelength holes in a metal film can be enhanced by the intentional presence of dyes in the system. As the transmission maximum occurs spectrally close to the absorption resonances of the dyes, this phenomenon was christened "absorption induced transparency". Here, a theoretical study on absorption induced transparency is presented. The results show that the appearance of transmission maxima requires that the absorbent fills the holes and that it occurs also for single holes. Furthermore, it is shown that the transmission process is nonresonant, being composed by a sequential passage of the electromagnetic field through the hole. Finally, the physical origin of the phenomenon is demonstrated to be nonplasmonic, which implies that absorption induced transparency should also occur at the infrared or terahertz frequency regimes.
\end{abstract}

DOI: 10.1103/PhysRevB.88.155126

PACS number(s): 42.25.Bs, 42.70.Jk, 78.20.Bh, 78.67.-n

The transmission of light through apertures has been attracting the attention of mankind for centuries. For instance, it was used in the invention of the camera obscura, it was at the root of the discussions on whether light has a corpuscular or wave nature, and nowadays it is central to the technique of optical lithography. A limiting factor for many applications is that the transmission process is severely impaired if the lateral hole dimension is smaller than the wavelength of light. ${ }^{1}$ In the last 15 years, several instances have been found in which light transmission through small holes is enhanced: (i) in arrays of subwavelength holes ${ }^{2}$ and single holes surrounded by surface corrugations, ${ }^{3}$ where light scattered by each hole, instead of being reflected, couples to surfaces modes, which allows the buildup of constructive interferences, ${ }^{4,5}$ (ii) through localized resonances in single subwavelength holes, ${ }^{6,7}$ occurring close to the cutoff frequency of the hole, which can be seen either as a zero-order Fabry-Perot resonance ${ }^{8}$ or transmission in an epsilon-near-zero material; ${ }^{9}$ and (iii) for Brewster-angle transmission, ${ }^{10}$ appearing at oblique illumination when the holey surface is impedance matched to the medium of incidence. All these phenomena are now generically known as extraordinary optical transmission (EOT). ${ }^{11}$ Recently, another case of EOT has been unveiled: absorption induced transparency (AIT). ${ }^{12}$ Roughly speaking, AIT refers to an enhancement in the transmittance through hole arrays that appears when an absorbing dye is deposited on them. The most intriguing characteristic of AIT is that the transmission peak, in the combined hole array plus dye system, appears at the spectral position where the bare dye presents resonant absorption. Several mechanisms have been proposed, such as dipole-induced dipole interaction between molecules and localized surface plasmon polaritons (SPPs), cutoff function modification, changes in Fresnel's coefficients, ${ }^{12}$ and coherent interactions between the hybridized fields of the oscillating elements present in the system, ${ }^{13}$ but the origin of AIT still remains unclear.
In this work we elucidate the physical origin of AIT, which we ascribe mainly to the change in the propagation constant inside the holes when filled by the dye. This is originated by the highly dispersive optical response of the dye at frequencies close to the absorption band. In particular, AIT transmission maxima (minima) very closely coincide with minima (maxima) in the imaginary part of the propagation constant of the hole. We predict that also single holes should exhibit AIT. We also show that the effect does not have a plasmonic origin and it is thus not restricted to metal films in the optical regime.

In this paper the electromagnetic (em) fields (and from them the reflectance, transmittance, and absorbance spectra) are computed using the finite difference time domain (FDTD) method $^{14}$ and, more particularly, the implementation to study silver films described in Ref. 15.

Let us first analyze the experimental results on AIT, reported for square arrays of circular holes carved on a silver film, placed on a glass substrate, and illuminated at normal incidence. ${ }^{12}$ The film thickness is $h=200 \mathrm{~nm}$, and the period $p=250 \mathrm{~nm}$ [see schematics in Fig. 1(b)]. These parameters will be kept fixed throughout this work. The optical response of the dye (which hereafter will be denoted as the absorber) used in the experiments is described by a frequency-dependent dielectric constant $\varepsilon_{A}(\omega)$. This classical electrodynamics approach is fully justified by the high molecular concentration levels and low laser powers used in experiments. ${ }^{16,17}$

In order to implement a realistic model with a minimum of material parameters, we consider that $\varepsilon_{A}$ is characterized by a single Lorentzian term: $\varepsilon_{A}(\omega)=\varepsilon_{h}-\Delta \varepsilon \Omega^{2} /\left(\omega^{2}-\Omega^{2}+\right.$ $\iota \omega \Gamma)$. The different parameters are fitted to reproduce the main absorption peak appearing when an unperforated film is coated with a 30-nm-thick absorber (see Fig. 1 in Ref. 12). We obtain $\varepsilon_{h}=1.8$ (host medium), $\Delta \varepsilon=0.18, \Gamma=0.028 \mathrm{eV}$ (line width), and $\Omega=1.8 \mathrm{eV}$ (transition frequency). 


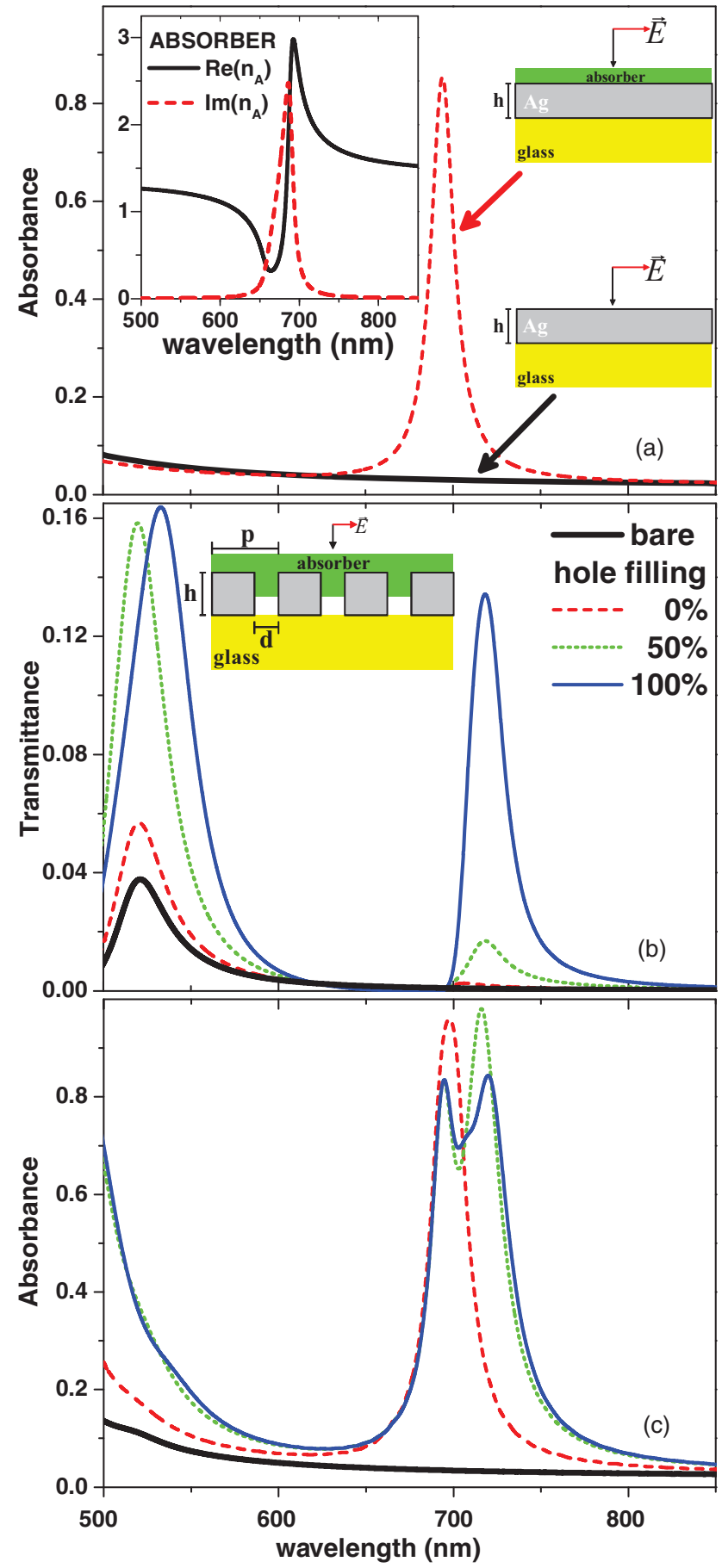

FIG. 1. (Color online) (a) Absorption spectra for an optically thick silver film, illuminated at normal incidence from the top interface. Two configurations are considered: (i) the silver film is coated with an absorbing overlayer (dashed line), with the refractive index represented in the inset (see main text for details), and (ii) no overlayer is present (solid line). (b) Transmittance through a square hole array of circular holes on a glass substrate for (i) no absorber present (thick solid line) and (ii) in the presence of an absorbing overlayer and different degrees of hole filling: no filling (dashed line), hole half filled (dotted line), and hole fully filled (thin solid line). (c) Absorbance for the same configurations as in (b). In all cases, the thickness of the absorbing layer is $30 \mathrm{~nm}$, the thickness of the metal film is $h=200 \mathrm{~nm}$, the period is $p=250 \mathrm{~nm}$, and the hole diameter is $d=140 \mathrm{~nm}$.
Figure 1(a) shows the absorption spectrum for the considered silver film, with and without the 30-nm-thick absorbing layer (dashed curve and solid curve, respectively). As expected, light is mainly reflected by the bare flat silver film, with an absorbance spectrum showing a smoothly decreasing trend as the wavelength increases. The presence of the thin absorber layer induces a large absorption peak, which is correlated with the resonant behavior in the imaginary part of the absorber refractive index, $\operatorname{Im}\left(n_{A}\right)$, represented in the inset of Fig 1(a).

Figure 1(b) renders the transmittance spectra for a hole array (with 140-nm-diameter circular holes) on a glass substrate, for different configurations of the absorber. The thick solid line renders the transmittance for the case in which no absorber is present, showing an EOT peak at $\lambda \sim 525 \mathrm{~nm}$. At this wavelength the waveguide modes inside the hole are evanescent (the cutoff wavelength for the fundamental mode is $\lambda_{c}^{o} \sim 430 \mathrm{~nm}$ ), and the EOT peak originates from the gratingassisted resonant excitation of surface electromagnetic modes at the glass-metal interface ${ }^{2,4}$ (SPPs in the optical regime). The other configurations analyzed in Fig. 1(b) have a 30-nm absorber thin overlayer and different degrees of hole filling: no absorber inside the hole (dashed line), absorber filling 50\% of the hole length (dotted line), and holes completely filled (thin solid line). The addition of the absorber induces two apparent changes to the transmittance spectra. First, the EOT peak at $\lambda \sim 525 \mathrm{~nm}$ is slightly increased by the presence of the overlayer (due to the stronger binding of the SPP to the surface and the corresponding enhancement of the SPP-hole coupling) and more strongly boosted by the filling of the holes. In this case, the mechanism behind is the hybridization between SPPs and localized resonances, ${ }^{6,18}$ which occurs near the cutoff wavelength. ${ }^{8,19}$ As $n_{A} \approx 1.2$ in the wavelength range from 500 to $600 \mathrm{~nm}$, the localized resonance is expected near $n_{A} \lambda_{c}^{o} \approx 515 \mathrm{~nm}$, thus overlapping with the SPP. The second change is, in agreement with the experimental finding, ${ }^{12}$ the appearance of the AIT peak at $\lambda \sim 720 \mathrm{~nm}$. Our calculations show that the intensity of the AIT peak strongly depends on the degree of filling of the holes: it is negligible when the absorber does not enter into the hole and maximum when the hole is completely filled. This transmittance peak is spectrally located close to the absorption line of the dye, but it is redshifted by $\sim 25 \mathrm{~nm}$ [in fact, a closer inspection reveals that the transmittance has a minimum very close to the maximum of $\left.\operatorname{Im}\left(n_{A}\right)\right]$. This shift is even more apparent in the absorbance, which is shown in Fig. 1(c) for the same systems considered in Fig. 1(b). This magnitude presents a double-peak structure in the AIT region. One absorption maximum occurs at the same spectral position as that of the absorbing overlayer, and, as the transmittance in the hole array is small there, it does not depend much on whether the holes are filled. The other absorption maximum coincides with the transmission peak; thus, it only occurs when the holes are at least partially filled.

AIT peaks can be related to the spectral features of the propagation constant of filled holes, $k_{z}$, which has an implicit analytical expression for circular waveguides. ${ }^{20}$ This is illustrated in Fig. 2, in which we focus on the AIT spectral region. We simultaneously represent the transmittance and reflectance for two hole arrays (characterized by different hole diameters) together with the imaginary and real part of 

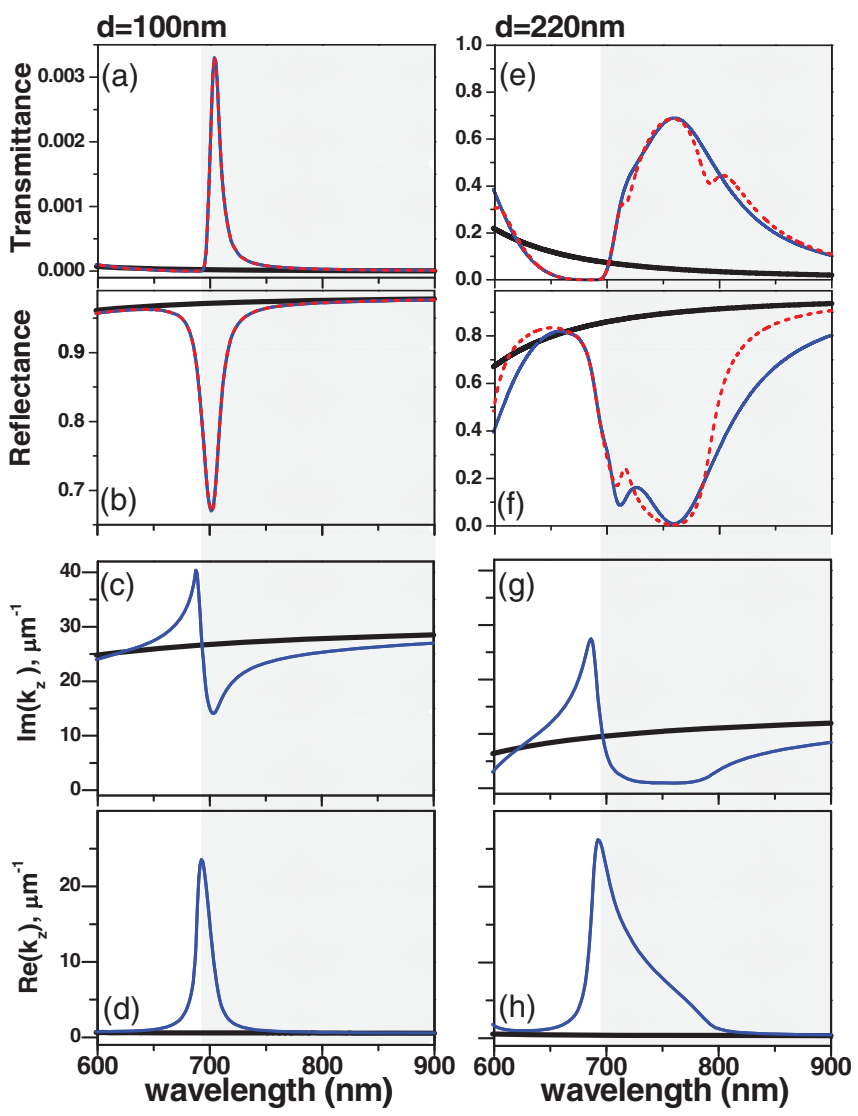

FIG. 2. (Color online) (a-d) Transmittance, reflectance, imaginary, and real part of the propagation constant inside the holes, respectively, for a free standing hole array with $p=250 \mathrm{~nm}$, $h=200 \mathrm{~nm}$, and $d=100 \mathrm{~nm}$. (e-h) Corresponding quantities for $d=220 \mathrm{~nm}$. Solid thick lines correspond to bare structures (without absorber), while thin solid lines are for filled holes. The dashed lines in the panels for transmittance and reflectance render the calculations neglecting multiple scattering (see main text for details).

$k_{z}$ inside the holes. In order to show that the presence of the substrate is not essential, the arrays are considered free standing (dielectric constant of substrate equal to unity). Both period $p=250 \mathrm{~nm}$ and film thickness $h=200 \mathrm{~nm}$ are as in the previous figures. In each panel, the thick continuous curves are for empty holes, while thin ones are for holes filled with the absorber (dashed lines will be discussed later on). The correlation between propagation constant and scattering coefficients shows that the enhancement of the transmission is due to a reduction in the imaginary part of $k_{z}$, and it indicates that AIT has a localized character. Notice also that transmittance peaks are narrower than reflection dips. This occurs because reflectance decreases when transmittance increases, but also when absorption increases, which occurs when the imaginary part of $k_{z}$ is enhanced (anomalous dispersion region).

A surprising result of the calculations is that AIT is not a resonant effect, at least in the range of geometrical parameters considered in the original experiments. This can be demonstrated by expressing the zero-order transmission $(t)$ and reflection $(r)$ amplitude coefficients through the whole structure in terms of the partial reflection coefficient at a single interface $(\rho)$. For a symmetric environment, the summation of multiple scattering processes gives $t=\left(1-\rho^{2}\right) e^{i k_{z} h} /(1-$ $\left.\rho^{2} e^{2 i k_{z} h}\right)$ and $r=\rho\left(1-t e^{i k_{z} h}\right)$. These expressions have been used in the past to analyze EOT phenomena. ${ }^{4,11,21}$ Resonant transmission is typically observed when the denominator in $t$ takes values close to zero. Of course, the previous expressions also apply to AIT, but in this case the multiple scattering terms can be neglected. This is readily done by considering that the reflection process only involves the first interface, so that $r \approx \rho$, while the transmission through the array is a three-step process: transmission into the hole, propagation inside it, and transmission out of the hole, leading to $t \approx\left(1-\rho^{2}\right) e^{i k_{z} h}$. The dashed red lines in the transmittance and reflectance panels in Fig. 2 are obtained using this approximation, with the coefficient $\rho$ computed numerically with the FDTD method. The excellent agreement with the full result confirms that AIT is not a resonant phenomenon in the sense of requiring the resonant buildup provided by multiple scattering. Instead, AIT transmission can be seen as a sequential process, favored by the reduced evanescence inside the holes induced by the absorber. This occurs at the long-wavelength side of the absorption resonance, where Kramers-Kronig relations provide a combination of large $\operatorname{Re}\left(n_{A}\right)$ and yet relatively small $\operatorname{Im}\left(n_{A}\right)$.

It is possible, following previous work on $\mathrm{EOT}^{11,22}$ and spoof plasmons, ${ }^{23}$ to develop an analytical minimal model for AIT. For that, the electromagnetic fields in different regions of space are expanded in the corresponding eigenfunctions, with expansion coefficients that are fixed by imposing the appropriate boundary conditions. Two approximations are then enforced: (i) only the least decaying mode inside the hole and the zero-order diffraction order in the radiation regions are considered and (ii) the properties of the metal are taken into account in such a way that the hole radius is phenomenologically enlarged by the actual skin depth; ${ }^{24}$ otherwise the metal is represented as a perfect electrical conductor (PEC, $\varepsilon_{\text {metal }}=$ $-\infty$ ). Within this simple model (which will be denoted MMPEC) it is straightforward to show that the perforated metal film with filled holes behaves as a uniform film characterized by a dielectric constant $\tilde{\varepsilon}=\left[\varepsilon_{A}-\left(\lambda / \lambda_{c}\right)^{2}\right] / S^{2}$ and an effective magnetic susceptibility $\tilde{\mu}=S^{2}$. In these expressions $S=$ $(\tilde{d} / p) \sqrt{\pi} / \sqrt{2\left(\gamma^{2}-1\right)}, \lambda_{c}=\pi \tilde{d} / \gamma$ is the cutoff wavelength of an unfilled circular waveguide in PEC, $\tilde{d}=d+2 \delta, \delta \approx$ $\lambda / 2 \pi \sqrt{\left|\operatorname{Re}\left(\varepsilon_{\mathrm{Ag}}\right)\right|} \approx 25 \mathrm{~nm}$ for silver in the optical regime, ${ }^{15}$ and $\gamma=1.848$. The inset to Fig. 3 shows, for an array of holes with diameter $d=140 \mathrm{~nm}$, the comparison between the exact result and that obtained with the minimal model for transmittance and also the real and imaginary parts of the propagation constant inside the film. The good agreement found for all these quantities validates the minimal model. This agreement is not restricted to the particular diameter considered. In Fig. 3 we represent the spectral position for both minimum and maximum AIT transmittance, together with the corresponding values for $\operatorname{Im}\left(k_{z}\right)$, computed both exactly and within the MMPEC model. These results confirm the correlation between transmittance and $\operatorname{Im}\left(k_{z}\right)$ discussed above. It also stresses that, as AIT does not have a plasmonic origin, it should also appear at shorter frequency regimes (e.g., THz or $\mathrm{mm}$ ), where the PEC approximation for the metal is even more accurate (of course, in this case, the holes should be filled by an absorber with an absorption line in the required frequency range). 


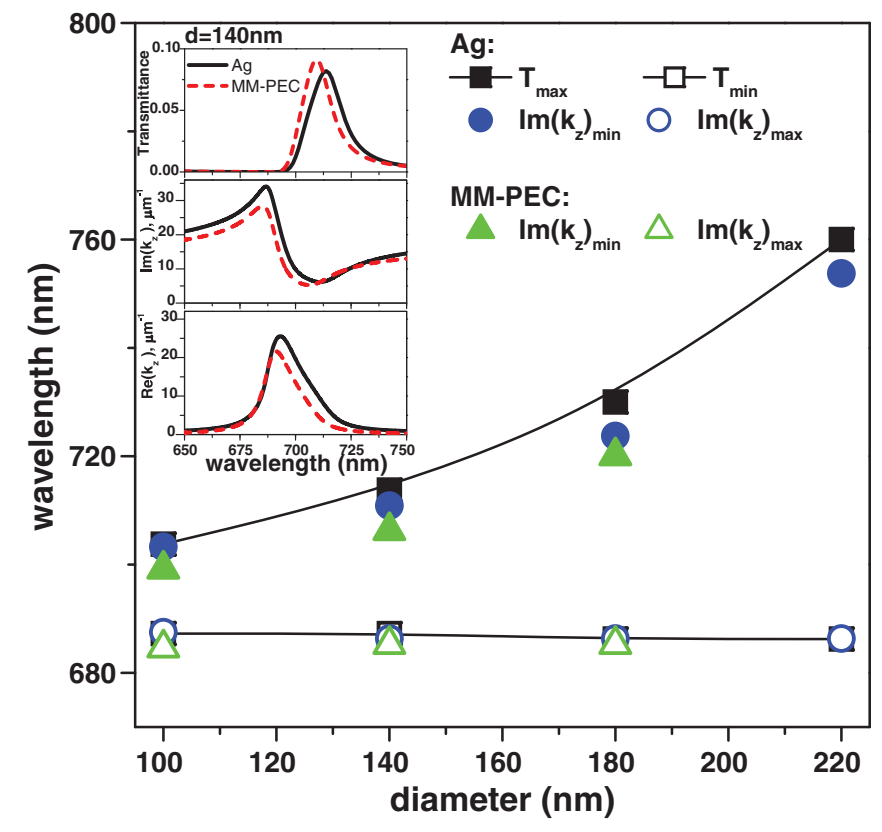

FIG. 3. (Color online) Spectral position of AIT transmittance maximum and minimum through a free standing hole array in a silver film, as a function of hole diameter (all other geometrical parameters are as in Fig. 2). The transmittance is represented by square symbols, which are filled in the case of maxima and empty for minima. Additionally, the spectral position for the maximum of $\operatorname{Im}\left(k_{z}\right)$ is shown with empty circular symbols, while that for the minimum is rendered by solid circles. The triangles represent $\operatorname{Im}\left(k_{z}\right)$ when the metal is considered to be a perfect electrical conductor. Inset: transmittance and $k_{z}$ for a hole array with $d=140 \mathrm{~nm}$. The considered metal is silver (solid lines) or PEC (dashed lines).

It could be argued that the experimentally demonstrated existence of AIT for hole arrays when the film is made of Ag but not when it is made of either $\mathrm{Ni}$ or $\mathrm{W}^{12}$ points to a plasmonic origin. This is not the case, as AIT is governed by the propagation constant inside the hole. This is illustrated in Fig. 4, which shows the maximum transmittance of the AIT peaks through fully filled hole arrays in different metals: $\mathrm{Ni}, \mathrm{W}$, and a series of hypothetical metals, where the real part of the dielectric constant is essentially that of $\mathrm{Ag}$ (so that the skin depth is practically the same in all the series), but the imaginary part is modified. The transmittance is represented as a function of $\operatorname{Im}\left(k_{z}\right)$ for the least evanescent mode inside the waveguide (evaluated at the spectral position of maximum transmittance). The good agreement with the estimation $T_{\max } \propto \exp \left[-2 \operatorname{Im}\left(k_{z}\right) h\right]$ (which depends only on the em properties inside the hole) shows that AIT in "bad" metals is hampered by the enhanced absorption inside the hole, and not by the existence, or lack of it, of surface electromagnetic modes at the metal surface.

Thus, the main physical mechanism involved in AIT, at least in the range of parameters experimentally considered, is the reduction in the opacity of the metal film due to the filling of the holes with a material with a large dielectric constant and moderate absorption (so that multiple scattering inside the hole is not relevant). The asymmetric shapes of the transmission peaks are, therefore, due to the spectral asymmetry of the real part of the refractive index close to a resonance, and not

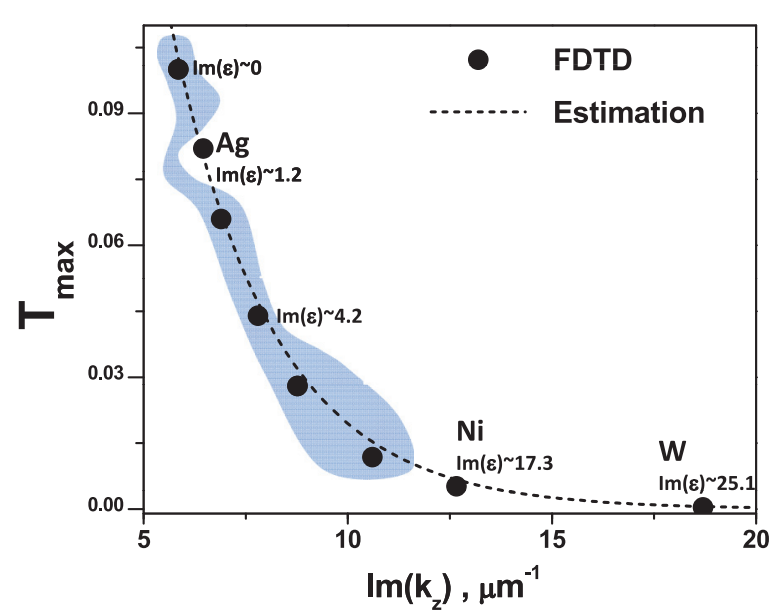

FIG. 4. (Color online) Maximum transmittance of the AIT peaks through a hole array of circular holes in a film made with different materials (period $p=250 \mathrm{~nm}$, film thickness $h=200 \mathrm{~nm}$, and hole diameter $d=140 \mathrm{~nm}$ ) as a function of $\operatorname{Im}\left(k_{z}\right)$, for the least evanescent mode inside the waveguide (evaluated at the spectral position of the maximum transmittance). The data points are obtained from FDTD calculations, for films made of either $\mathrm{W}, \mathrm{Ni}$, or a series of hypothetical metals (points in the shaded area), where the real part of the dielectric constant is essentially that of Ag but the imaginary part is modified. The discontinuous curve is obtained from $T_{\max }=C \exp \left[-2 \operatorname{Im}\left(k_{z}\right) h\right]$, where the constant $C$ is fixed so that the curve crosses the data point for $\operatorname{Ag}[\operatorname{Im}(\varepsilon) \sim 1.2]$.

to any "Fano-like" resonant effect. This conclusion is further supported by analyzing the maximum transmission for arrays of partially filled holes (where the hole is filled a distance

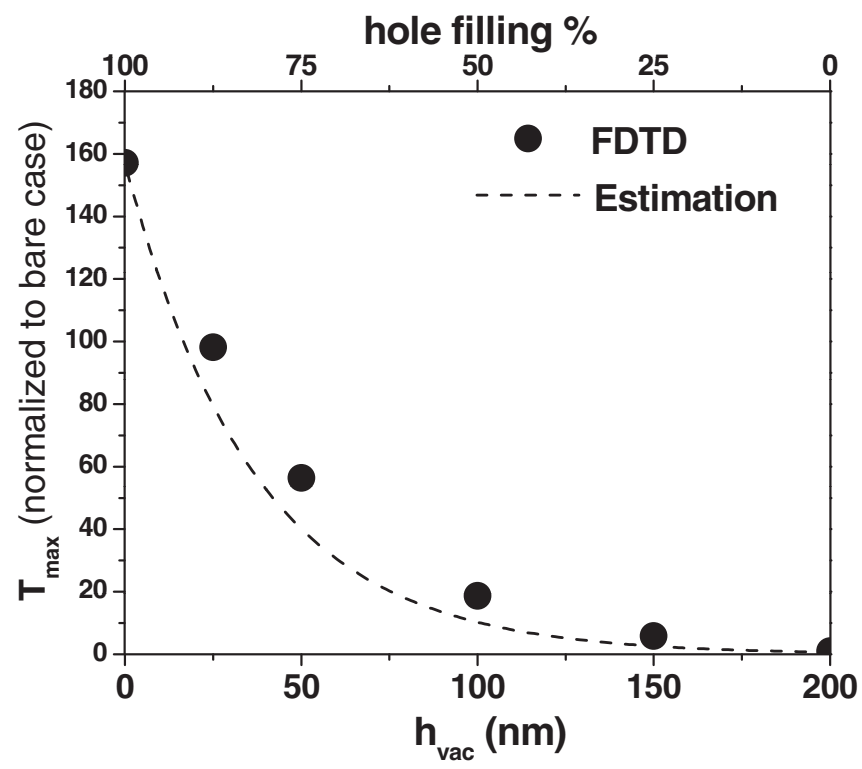

FIG. 5. Maximum transmittance of the AIT peaks (normalized to the bare case transmittance at the corresponding wavelength) in a hole array of circular holes in silver (period $p=250 \mathrm{~nm}$, film thickness $h=200 \mathrm{~nm}$, and hole diameter $d=140 \mathrm{~nm}$ ). The holes are filled by the dye a distance $h-h_{\text {vac }}$ from the top interface and unfilled a distance $h_{\text {vac }}$ from the bottom interface. Solid points represent the results obtained from full FDTD calculations, while the discontinuous line is obtained by a model where the transmission process is assumed to be sequential (see main text for details). 

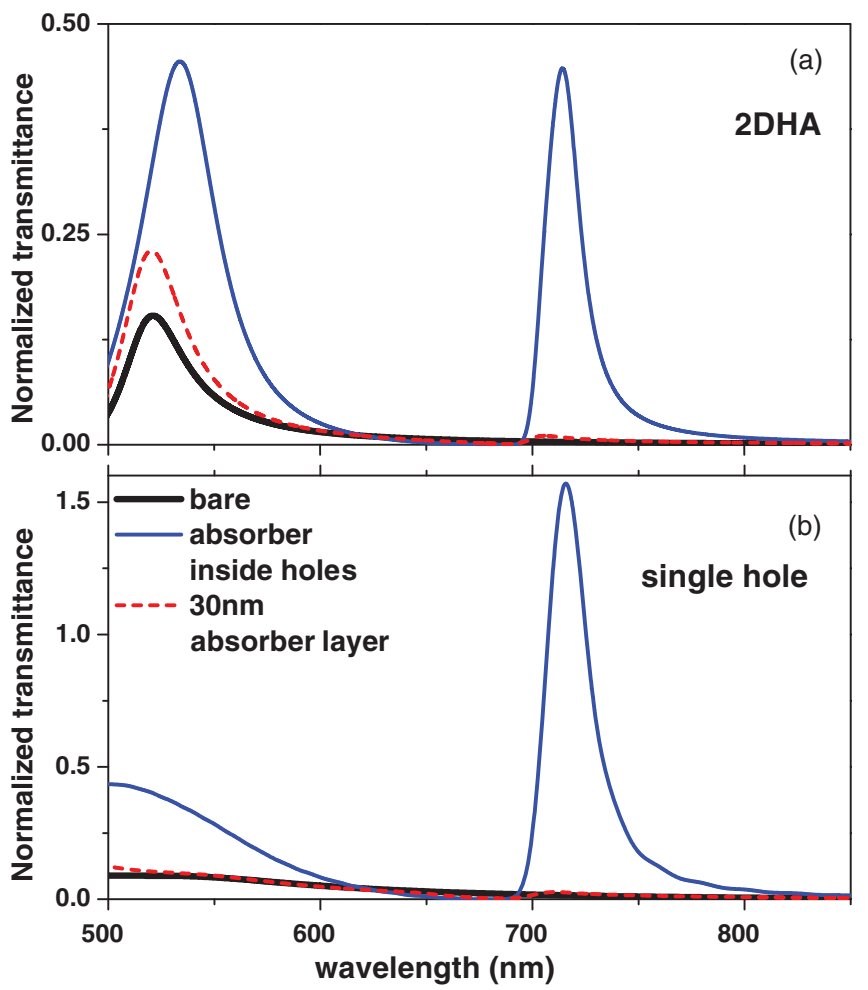

FIG. 6. (Color online) (a) Transmittance through a hole array of circular holes in silver (period $p=250 \mathrm{~nm}$, film thickness $h=200 \mathrm{~nm}$, and hole diameter $d=140 \mathrm{~nm}$ ). The thick solid line represents the bare hole array, the thin solid line represents the holes filled with an absorber, and the thin dashed line represents holes empty but with the array coated with a 30-nm absorbing layer. (b) Transmittance through a single hole for the same cases as in panel (a). In both cases the transmittance has been normalized to the hole area.

$h-h_{\text {vac }}$ from the top surface and unfilled otherwise). Figure 5 shows, together with the FDTD results, the curve $T_{\max }\left(h_{\mathrm{vac}}\right)=$ $T_{\max }(0) \exp \left[-2 \operatorname{Im}\left(k_{z}^{\mathrm{vac}}-k_{z}\right) h_{\mathrm{vac}}\right]$. This expression is obtained by assuming that the transmission through the structure is a sequential process, in which the propagation in each region is dominated by the corresponding propagation constant $\left(k_{z}^{\mathrm{vac}}\right.$ in the un-filled hole region and $k_{z}$ in the filled one). Again, the good agreement between the full calculations and this extremely simplified model indicates that the main factor governing AIT is the modification of the propagation constant.

It is also interesting to study whether AIT also occurs for isolated holes. In Fig. 6 we compare the transmittance through both a hole array (top panel) and a single hole (bottom panel). The period, metal thickness, hole diameter, and substrate are as in the case considered in Fig. 1. We analyzed three different configurations: no absorber, a 30-nm absorber overlayer with empty holes, and fully filled holes without an overlayer. These calculations confirm the hybrid nature of the EOT peak appearing at $\sim 525 \mathrm{~nm}$, which for hole arrays already occurs when no dye is present, while in a single hole it only occurs when the dye fills the hole and induces the presence of localized resonances. Regarding AIT, the fact that, for both single holes and hole arrays, the peak emerges only when holes are filled confirms that AIT is a localized effect, where collective interactions between holes are not essential.

To conclude, we have demonstrated that AIT requires the presence of absorbers, like molecules embedded in a polymer, inside the holes of a perforated metal film. Our calculations predict that AIT should also occur in single holes, having thus a localized character. We have found that their spectral position and width, and their intensity, are mainly controlled by the imaginary part of the propagation constant of holes. We have demonstrated that hole arrays in the AIT regime behave like a metamaterial, characterized by a dielectric constant composed by a Drude plasma term (of geometric origin) plus a Lorentz term arising by the presence of the absorber. We have shown that AIT peaks are nonplasmonic in character, so they are predicted to occur in frequency regimes other than the optical. This opens the door for detection spectroscopy of chemical compounds characterized by sharp absorption lines in the $\mathrm{THz}$ or microwave regimes.

We acknowledge support from the Spanish Ministry of Science and Innovation under Projects No. MAT2011-28581-C02 and No. CSD2007-046-Nanolight.es. F.J.G.-V. acknowledges financial support from the European Research Council under Grant No. 290981 (PLASMONANOQUANTA). *sergut@unizar.es

${ }^{1}$ H. A. Bethe, Phys. Rev. 66, 163 (1944).

${ }^{2}$ T. W. Ebbesen, H. L. Lezec, H. F. Ghaemi, T. Thio, and P. A. Wolff, Nature (London) 391, 667 (1998).

${ }^{3}$ H. J. Lezec, A. Degiron, E. Devaux, R. A. Linke, L. Martín-Moreno, F. J. García-Vidal, and T. W. Ebbesen, Science 297, 820 (2002).

${ }^{4}$ L. Martín-Moreno, F. J. García-Vidal, H. J. Lezec, K. M. Pellerin, T. Thio, J. B. Pendry, and T. W. Ebbesen, Phys. Rev. Lett. 86, 1114 (2001).

${ }^{5}$ H. Liu and P. Lalanne, Nature (London) 452, 728 (2008).

${ }^{6}$ K. J. K. Koerkamp, S. Enoch, F. B. Segerink, N. F. van Hulst, and L. Kuipers, Phys. Rev. Lett. 92, 183901 (2004).

${ }^{7}$ A. Degiron, H. J. Lezec, N. Yamamoto, and T. W. Ebbesen, Opt. Commun. 239, 61 (2004).

${ }^{8}$ F. J. García-Vidal, E. Moreno, J. A. Porto, and L. Martín-Moreno, Phys. Rev. Lett. 95, 103901 (2005).
${ }^{9}$ M. Silveirinha and N. Engheta, Phys. Rev. Lett. 97, 157403 (2006).

${ }^{10}$ A. Alù, G. D'Aguanno, N. Mattiucci, and M. J. Bloemer, Phys. Rev. Lett. 106, 123902 (2011).

${ }^{11}$ F. J. Garcia-Vidal, L. Martin-Moreno, T. W. Ebbesen, and L. Kuipers, Rev. Mod. Phys. 82, 729 (2010).

${ }^{12}$ J. A. Hutchison, D. M. O'Carroll, T. Schwartz, C. Genet, and T. W. Ebbesen, Angew. Chem. Int. Ed. 50, 2085 (2011).

${ }^{13}$ P. Weis, J. L. Garcia-Pomar, R. Beigang, and M. Rahm, Opt. Express 19, 23573 (2011).

${ }^{14}$ A. Taflove and S. C. Hagness, Computational Electrodynamics: The Finite-Difference Time-Domain Method, 3rd ed. (Artech House, Boston, 2005).

${ }^{15}$ S. G. Rodrigo, F. J. García-Vidal, and L. Martín-Moreno, Phys. Rev. B 77, 075401 (2008).

${ }^{16}$ U. C. Fischer, E. Bortchagovsky, J. Heimel, and R. T. Hanke, Appl. Phys. Lett. 80, 3715 (2002). 
${ }^{17}$ F. Valmorra, M. Bröll, S. Schwaiger, N. Welzel, D. Heitmann, and S. Mendach, Appl. Phys. Lett. 99, 051110 (2011).

${ }^{18}$ A. Degiron and T. W. Ebbesen, J. Opt. A: Pure Appl. Opt. 7, S90 (2005).

${ }^{19}$ S. Carretero-Palacios, F. J. García-Vidal, L. Martín-Moreno, and S. G. Rodrigo, Phys. Rev. B 85, 035417 (2012).

${ }^{20}$ J. D. Jackson, Classical Electrodynamics, 2nd ed. (Wiley, New York, 1975).
${ }^{21}$ F. J. $\quad$ G. de Abajo, Rev. Mod. Phys. 79, 1267 (2007).

${ }^{22}$ L. Martín-Moreno and F. J. García-Vidal, J. Phys.: Condens. Matter 20, 304214 (2008).

23 J. B. Pendry, L. Martín-Moreno, and F. J. García-Vidal, Science 305, 847 (2004).

${ }^{24}$ L. Martín-Moreno and F. J. García-Vidal, Opt. Express 12, 3619 (2004). 